Instituto de Terapéutica Experimental y Clínica, del Departamento de Fisiología del Centro Universitario de Ciencias de la Salud de la Universidad de Guadalajara. Guadalajara, México. ${ }^{\mathrm{a} M S c}$. bPhD.

'MD.

Recibido el 24 de mayo de 2017, aceptado el 30 de enero de 2018.

Trabajo no recibió financiamiento. Los autores no declaran conflictos de interés.

Correspondencia a: Carlos Gerardo Ramos Becerra Sierra Mojada 950, edificio P, $1^{\circ}$ piso, colonia Independencia. C.P 44340 Guadalajara Jalisco, México. Teléfono: (33) 10585200 ext. 34215,34111 . Fax (33) 36173490 md_crb@hotmail.com

\section{Confiabilidad de un monitor automatizado para la medición de la presión arterial}

\author{
DAVID CARDONA-MÜLLER ${ }^{\mathrm{a}}$, FERNANDO GROVER-PÁEZ ${ }^{\mathrm{b}}$, \\ VÍCTOR GUZMÁN-SALDIVAR ${ }^{c}$, GUILLERMO A. ALANIS-SÁNCHEZc ${ }^{c}$, \\ CÉSAR MURGUIIA-SOTO, SYLVIA E. TOTSUKA-SUTTO ${ }^{\mathrm{b}}$, \\ PATRICIA QUEZADA-FERNÁNDEZ ${ }^{c}$, AGUSTÍN MACÍAS-CHUMACERA ${ }^{c}$, \\ ERNESTO G. CARDONA-MUÑOZ ${ }^{\text {b }}$, CARLOS G. RAMOS-BECERRA ${ }^{b}$

\section{Reliability of an automatic monitor for blood pressure measurement}

Background A correct blood pressure (BP) measurement is essential for the diagnosis and control of high BP. Aim: To evaluate the agreement and repeatability of BP measurements with the OMRON HEM-7320-LA device compared to a mercury sphygmomanometer. Material and Methods: A cross-sectional study comparing BP measurements made by two randomly selected trained nurses and an automatic oscillometric device. The mercurial sphygmomanometer was connected to the automated device via a " $T$ " type connector and a dual-head stethoscope was used, allowing simultaneous measurements. The results were analyzed with one-factor analysis of variance, Bland-Altman's test, repeatability coefficient (RC), and intra-class correlation coefficient (ICC). Results: Forty-nine participants aged $56 \pm 19$ years were included. Nineteen had hypertension (38\%). We did not observe a significant difference in either systolic $(S B P)$ or diastolic blood pressure $(D B P)$ pressure measurements between the observers and the device. The mean difference was $-0.09 \mathrm{mmHg}(95 \%$ confidence intervals (CI)-0.9 to 0.7) for SBP and $-0.9 \mathrm{mmHg}$ (95\% CI - 1.7 to -0.13) for DBP. The RC for $\operatorname{SBP}(6.2,5.2$ and $5.8 \mathrm{mmHg})$ and $D B P(4.7,4.2$ y $5.2 \mathrm{mmHg}$ ) was similar between the observers and the device. The ICC for SBP was 0.990 (95\% CI 0.983 to 0.995, $p<0.01)$ and 0.986 (95\% CI 0.977 to 0.991, $p<0.01)$ for DBP. Conclusions: There was a high level of agreement and similar measurement repeatability in the measurements performed by the automatic device and the mercurial sphygmomanometer. No differences in BP measurements were observed.

(Rev Med Chile 2018; 146: 190-195)

Key words: Blood Pressure; Blood Pressure Determination; Hypertension.
A nivel mundial existe un aumento en la prevalencia de las enfermedades cardiovasculares ${ }^{1}$. Anualmente ocurren aproximadamente ocho millones de muertes a nivel mundial atribuibles a complicaciones con la hipertensión arterial (HTA). El inadecuado control de HTA es de los principales factores de riesgo de eventos vasculares cerebrales e infartos al miocardio a nivel mundial, incluyendo a Méxi$\mathrm{CO}^{2-5}$, y a su vez es responsable de un incremento de los costos en salud pública en los países en vías de desarrollo ${ }^{6}$. 
La Organización Mundial de la Salud (OMS) ha identificado la reducción del riesgo cardiovascular total a través del control y tratamiento integrado de los factores de riesgo, como la hipertensión, como una de las estrategias más eficaces para hacer frente a la epidemia mundial de enfermedades cardiovasculares ${ }^{7}$. También ha reconocido que una de las principales causas de un mal control de la Presión Arterial (PA) es la falta de disponibilidad de dispositivos fiables, fáciles de obtener, y asequibles para la medición de la PA, un problema que probablemente se incrementará a medida que los esfigmomanómetros de mercurio sean retirados.

Además, el problema se agrava por la comercialización de aparatos para la medición de la PA que no han sido validados, el alto costo global de los dispositivos, y una escasez de trabajadores de la salud tanto médicos como no médicos entrenados para la medición de PA de acuerdo a las guías internacionales. La popularidad y el uso de los dispositivos oscilométricos automáticos para la medición de la PA han aumentado en los últimos años, especialmente para la automedición en casa y en algunos centros hospitalarios, así como clínicas de atención primaria. A pesar de ello, existe la percepción entre el personal de enfermería, incluso en el personal médico que la confiabilidad de los valores obtenidos mediante estos equipos electrónicos es muy cuestionable respecto a los obtenidos con tensiómetros aneroides o mediante el estándar de oro que es el tensiómetro mercurial. El objetivo de la presente investigación fue evaluar la confiabilidad entre las mediciones realizadas con el aparato OMRON ${ }^{\circledR}$ HEM-7320 versus las obtenidas con el esfigmomanómetro de mercurio obtenidas por el personal de enfermería en la práctica clínica en un primer nivel de atención médica.

\section{Material y Métodos}

Para el cálculo del tamaño de la muestra, se utilizó la fórmula para estimar diferencia de medias en una población finita, cuya variable principal fue de tipo cuantitativa, con un nivel de confianza de $95 \%$ y un poder estadístico de $80 \%$. Se incluyeron pacientes que acudieron a consulta a una unidad médica de primer contacto del Sector Salud, Guadalajara, Jalisco, México.
Todos los procedimientos y actividades que fueron realizadas a lo largo del estudio se respetaron de acuerdo a las normas éticas de acuerdo a la declaración de Helsinki versión 2013, y las guías de buena práctica clínica. Se solicitó la participación mediante carta de consentimiento bajo información por escrito de todos los participantes. El protocolo de investigación, así como el consentimiento bajo información fueron sometidos, revisados y aprobados por el comité de ética en investigación del Centro Universitario de Ciencias de la Salud, Universidad de Guadalajara.

A todos los pacientes se les determinaron las cifras de presión arterial sistólica (PAS) y presión arterial diastólica (PAD), conforme a la Norma Oficial Mexicana NOM-030-SSA2-2009, para la prevención, detección, diagnóstico, tratamiento y control de la hipertensión arterial sistémica ${ }^{8}$.

Para la medición de la presión arterial se utilizó el aparato automático oscilométrico OMRON ${ }^{\circledR}$ modelo HEM-7320-LA (versión para América Latina). El cual ha sido validado y cumple con los criterios establecidos por la Sociedad Europea de Hipertensión en su revisión de 2010, y es recomendado para su uso en la población en general $^{9}$, y un esfigmomanómetro de mercurio de mesa certificado y validado marca Riester $^{\circledR}$ Modelo 1010-108. Ambos dispositivos fueron calibrados antes de realizar las mediciones. El estudio incluyó dos enfermeras del departamento de medicina preventiva elegidas en forma aleatoria y un médico general que fungió como supervisor, los cuales fueron entrenados en la correcta toma de la PA de acuerdo a las guías de la ESH (por sus siglas en inglés European Society of Hypertension $)^{10}$. Tanto las enfermeras como el supervisor fueron cegados a los resultados de las tomas de PA realizadas por cada uno de ellos. Se midió la circunferencia braquial de cada paciente para utilizar el brazalete adecuado, con las siguientes dimensiones: mediano (circunferencias $22-32 \mathrm{~cm}$ ) y grande (circunferencias $32-42 \mathrm{~cm}$ ). La medición de la PA se realizó de forma simultánea entre el supervisor que operó el equipo oscilométrico $\mathrm{OMRON}^{\circledR}$ modelo HEM-7320-LA y las dos enfermeras que utilizaron el esfigmomanómetro de mercurio. Para lograr una sincronización en la auscultación, se modificó el monitor automatizado. La salida de aire emitida por el compresor se derivó mediante un conector plástico de dos vías en " $\mathrm{T}$ ", una al brazalete del paciente y la otra a la 
columna de mercurio del esfigmomanómetro. Se utilizó un estetoscopio de doble auricular de marca Littman ${ }^{\circledR}$ Cardiology para así garantizar la medición simultánea entre los 2 observadores. Se llevaron a cabo tres mediciones en cada paciente con espacio de un minuto entre tomas.

El análisis estadístico se llevó a cabo con el programa SPSS v20 (SPSS, Chicago, IL, USA). Los valores obtenidos se expresan en medias y desviación estándar. Para determinar la diferencia de los valores obtenidos, se compararon los promedios de las tres mediciones entre los observadores mediante el análisis de varianza de un factor. Para evaluar la concordancia se utilizó método de Bland-Altman, en el cual compararon el promedio de las mediciones de los observadores contra las mediciones del equipo. Así también, se evaluó la repetitividad de las mediciones de cada observador y el equipo, calculando la desviación estándar (DE) intra-sujeto de la segunda y tercera medición, y posteriormente su coeficiente de repetitividad como $1,96 x \sqrt{ } 2$ multiplicado por la DE intra-sujeto ${ }^{11}$. El cual representa el valor por debajo del cual la diferencia absoluta de dos mediciones consecutivas, caerá en $95 \%$ de las tomas ${ }^{11}$. Además, para determinar la concordancia de los valores obtenidos por el instrumento y los observadores, se determinó el coeficiente de correlación intraclase (CCI) para observadores fijos. El CCI se mide en una escala de 0 a 1 , el cual se define como la proporción de la variabilidad total debida a la variabilidad de los pacientes ${ }^{12}$. Se tomó una $\mathrm{p}<0,05$ a dos colas como significancia estadística. El tamaño de la muestra se estimó utilizando el software $G^{\star}$ Power (versión
3.1.9.2 $)^{13}$ con un nivel de significancia $(\alpha)$ de 0,05 y un poder estadístico de 0,90 resultando en 48 participantes por grupo.

\section{Resultados}

Se realizó la medición de la PA en 49 pacientes. La edad promedio fue 56,3 $\pm 17,8$ años, 19 (38\%) estaban catalogados como hipertensos, $42(85 \%)$ fueron mujeres, ningún paciente refirió tabaquismo previo o actual, ni otras comorbilidades asociadas. En la Tabla 1 se muestran los promedios de las tres mediciones de la PAS y PAD obtenidas por el observador 1, 2 y equipo. Se compararon los promedios inter-observador para la PAS y PAD de los observadores y el equipo. No se observó diferencia significativa en la primera, segunda o tercera medición de PAS ( $\mathrm{p}>0,05)$, así como tampoco se observó en la PAD ( $\mathrm{p}>0,05)$.

Para evaluar la concordancia entre las cifras de los observadores y el equipo, se aplicó la prueba de Bland-Altman (Figura 1), con sesgo de -0,09 (95\% IC de -0,9 a 0,7). Los límites de concordancia (LC) de $95 \%$ fueron de $-5,5$ a 5,3. Sólo dos mediciones salieron de los LC, haciendo la precisión de las mediciones aceptable para la mayoría de los sujetos. En cuanto a la PAD el sesgo de medición fue de $-0,9 \mathrm{mmHg}$ (95\% IC de -1,7 a -0,1). Sus LC de 95\% fueron de $-6,4$ a $4,5 \mathrm{mmHg}$ con dos mediciones saliendo de estos límites (4\%).

El coeficiente de repetibilidad para la PAS del observador 1 fue 6,2 mmHg, observador 2 de 5,2 $\mathrm{mmHg}$ y el dispositivo de 5,8 $\mathrm{mmHg}$. Así también, para la PAD fue de $4,7 \mathrm{mmHg}, 4,2 \mathrm{mmHg}$ y 5,2

Tabla 1. Análisis de varianza de un factor entre los diferentes observadores y el dispositivo oscilométrico

\begin{tabular}{|cccccc|}
\hline & OB 1 & OB 2 & Equipo & F & p \\
\hline TA Sistólica & & & & 0,916 \\
Medición 1 & 127,5 & 125,9 & 127,1 & 0,08 & 0,984 \\
Medición 2 & 125,7 & 126,2 & 126,3 & 0,01 & 0,930 \\
Medición 3 & 125,8 & 124,5 & 124,6 & 0,07 & 0,608 \\
TA Diastólica & & & & 0,50 & 0,807 \\
Medición 1 & 78,4 & 80,8 & 80,5 & 0,21 & 0,736 \\
Medición 2 & 78,0 & 79,3 & 78,9 & 0,30 & 77,4 \\
Medición 3 & 76,6 & 78,5 & & & \\
\hline
\end{tabular}

OB 1: Observador 1; OB 2: Observador 2. 


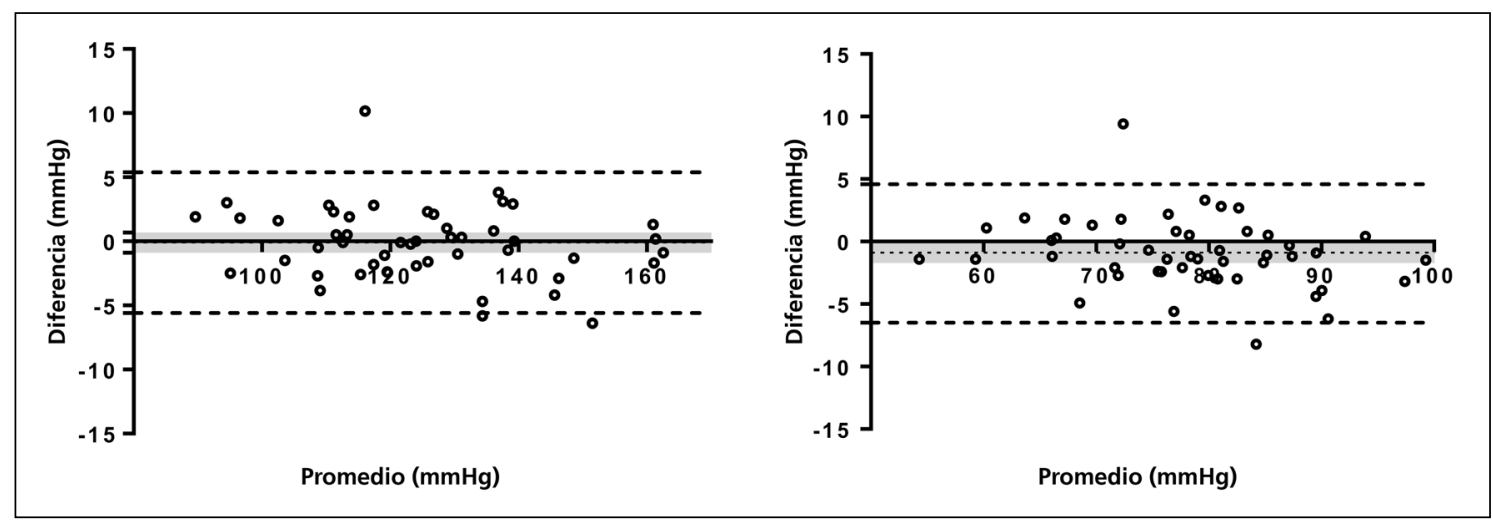

Figura 1. Gráfico de la diferencia (eje y) de la presión arterial sistólica (izquierda) y diastólica (derecha) vs el promedio (eje x) de las mediciones de los observadores y el equipo. Los valores son de los 49 pacientes evaluados. Las líneas punteadas externas indican a los límites de concordancia. El área sombreada representa los intervalos de confianza de la diferencia media.

Tabla 2. Diferencia media de las Deltas entre observadores y equipo

\begin{tabular}{|ccccc|}
\hline & Delta OB 1 vs OB 2 & Delta OB 1 vs Equipo & Delta OB 2 vs Equipo & p* $^{*}$ \\
\hline PAS & $0,75(2,73)$ & $0.30(3.42)$ & $-0,44(2,45)$ & 0,07 \\
\hline PAD & $-0,42(2,49)$ & $-1.16(3.1)$ & $-0,74(3)$ & 0,30 \\
\hline
\end{tabular}

OB 1: Observador 1; OB 2: Observador $2 .{ }^{*}=$ ANOVA de medidas repetidas.

$\mathrm{mmHg}$, respectivamente. El CCI de observadores fijos del promedio de mediciones de PAS fue de 0,990 (95\% IC de 0,983 a 0,995; p < 0,001) y de $0,986$ (95\% IC de 0,977 a 0,$991 ; \mathrm{p}<0,001)$ para la PAD indicando una alta confiabilidad ${ }^{12}$ de las mediciones entre dispositivo y los observadores.

\section{Discusión}

La medición correcta de la PA es el elemento principal para el diagnóstico, pronóstico, estratificación de riesgo cardiovascular y decisión terapéutica en los pacientes con HTA. Existen errores comunes en la toma de la presión arterial que pueden estar determinados por el tipo de equipo, sesgos en la medición por el observador y las condiciones ambientales donde se realiza el procedimiento. El uso de equipos automatizados para la medición de la presión PA puede eliminar los errores generados por los sesgos de medición de quien toma la presión y en buena parte el ambiental. Sin embargo, la gran variedad de equipos comercializados, han generado la percepción entre los usuarios que algunos de éstos son poco confiables. El presente estudio evaluó la confiabilidad del uso del equipo automatizado OMRON HEM-7320-LA, para la medición de la PA en escenario controlado, en una clínica de primer contacto, al compararlo con cifras de PA obtenidas por el personal de enfermería que usó el estándar de oro (esfigmomanómetro de mercurio).

No se identificaron diferencias significativas entre los valores de PAS y PAD obtenidos por los observadores comparados con los obtenidos por el equipo automatizado. Las diferencias observadas fueron pequeñas, con diferencia media menor de $2 \mathrm{mmHg}$, que se encuentra dentro del rango de una diferencia aceptable para un equipo confia$b^{14}{ }^{14}$. En la práctica diaria la PA es obtenida por diferentes personas y existe variación en la cifra de PA obtenida entre éstas. Por lo mismo se comparó si la variación entre el valor de la PA obtenida por equipo y personal de enfermería difiere en forma significativa con la variación presentada entre 2 
personas entrenadas para medir la PA, sin identificar diferencias significativas.

Esto es similar a lo reportado con otros equipos oscilométricos automáticos, Young-Hyo Lim et al. ${ }^{15}$ quienes investigaron el efecto que tenía el uso del dispositivo automatizado A\&D UA-767 para la toma de la PA en un sondeo epidemiológico en donde identificaron pequeñas diferencias entre la toma manual y automatizada de la presión arterial que repercute de forma significativa al momento de realizar el diagnóstico de HTA.

El uso de monitores automatizados para la medición de la PA es una estrategia útil para su medición en centros de primer contacto. Debido a su confiabilidad y disminución de errores en las mediciones con métodos convencionales (mercurio y aneroide) y con personal no capacitado para la toma correcta de la PA según las Guías Europeas de Hipertensión Arterial ${ }^{10}$.

A pesar de la pequeña diferencia observada entre el monitor OMRON HEM-7320-LA y el estándar de oro, se considera a este equipo una herramienta útil para el escrutinio y la medición de PA en centros de primer nivel. Ya que permite optimizar el tiempo del personal encargado de esta función y al mismo tiempo elimina algunos factores que favorecen la medición errónea de la PA, como es el factor humano y el ruido ambiental, entre otros.

Una de las fortalezas del presente estudio radica en la adaptación realizada al monitor automatizado para la medición de la PA. Esta adaptación permitió evaluar a los dos observadores y al monitor de forma simultánea eliminando así la variabilidad esperada entre tomas en los métodos tradicionalmente utilizados para validar/evaluar a los diferentes equipos. De igual forma, el estudio evalúa el desempeño del monitor automático para la medición de PA en una situación que se acerca a la realidad que se vive día a día en las clínicas de primer nivel en México. No obstante, el trabajo también tiene algunas debilidades, ya que el personal de enfermería encargado de realizar las mediciones fue entrenado para la medición correcta de la PA acorde a las recomendaciones de las guías mencionadas previamente. Sin embargo, esto dista de la práctica clínica cotidiana en las clínicas de primer contacto y hospitales, en donde la toma de la PA es un procedimiento rutinario importante, ya que de su correcta medición depende la toma de decisiones clínicas y terapéuticas.

\section{Conclusión}

Observamos una alta concordancia entre los datos obtenidos por el equipo de estudio y los observadores con la técnica de medición considerada como la estándar de oro, por lo tanto, el monitor automático para la medición de la presión arterial OMROM HEM-7320-LA, se considera confiable para la medición de la PA en sitios de atención primaria, siempre y cuando se cumplan con las recomendaciones de las guías de las distintas sociedades para el diagnóstico, control y tratamiento de la hipertensión arterial.

\section{Referencias}

1. Lawes CM, Vander Hoorn S, Rodgers A. Global burden of blood-pressure-related disease, 2001. Lancet 2008; 371 (9623): 1513-8.

2. Mendis S, Abegunde D, Oladapo O, Celletti F, Nordet P. Barriers to management of cardiovascular risk in a low-resource setting using hypertension as an entry point. Journal of hypertension 2004; 22 (1): 59-64.

3. Lawes CM, Vander Hoorn S, Law MR, Elliott P, MacMahon S, Rodgers A. Blood pressure and the global burden of disease 2000. Part II: estimates of attributable burden. Journal of hypertension 2006; 24 (3): 423-30.

4. Kearney PM, Whelton M, Reynolds K, Whelton PK, He J. Worldwide prevalence of hypertension: a systematic review. Journal of hypertension 2004; 22 (1): 11-9.

5. López AD, Mathers CD, Ezzati M, Jamison DT, Murray CJ. Global and regional burden of disease and risk factors, 2001: systematic analysis of population health data. Lancet 2006; 367 (9524): 1747-57.

6. Gaziano TA, Bitton A, Anand S, Weinstein MC. The global cost of nonoptimal blood pressure. Journal of hypertension 2009; 27 (7): 1472-7.

7. Bath P, Chalmers J, Powers W, Beilin L, Davis S, Lenfant C, et al. International Society of Hypertension (ISH): statement on the management of blood pressure in acute stroke. J Hypertens 2003; 21 (4): 665-72.

8. Mexicana NO. Para la prevención, detección, diagnóstico, tratamiento y control de la hipertensión arterial sistémica. NOM-030-SSA2-2009. 2009.

9. Grover-Páez F, Cardona-Muñoz EG, Cardona-Müller D, Guzmán-Saldívar VH, Rodríguez-De la Cerda M, Jiménez-Cázarez MB, et al. Validation of the Omron HEM-7320-LA, upper arm blood pressure monitor with Intelli Wrap Technology Cuff HEM-FL1 for self-measu- 
rement and clinic use according to the European Society of Hypertension International Protocol revision 2010 in the Mexican population. Blood pressure monitoring 2017; 22 (6): 375.

10. Mancia G, Fagard R, Narkiewicz K, Redon J, Zanchetti A, Böhm M, et al. ESH/ESC guidelines for the management of arterial hypertension: the Task Force for the Management of Arterial Hypertension of the European Society of Hypertension (ESH) and of the European Society of Cardiology (ESC). Blood pressure 2013; 22 (4): 193-278.

11. Institution BS. Precision of test methods, Part 1: Guide for the determination of repeatability and reproducibility for a standard test method London 1979.

12. Prieto L, Lamarca R, Casado A. La Evaluación De La Fiabilidad En Las Observaciones clínicas: el coeficiente de correlación intraclase. Med Clin (Barc) 1998; 110 (4): 142-5.

13. Faul F, Erdfelder E, Lang A-G, Buchner A. G* Power 3: A flexible statistical power analysis program for the social, behavioral, and biomedical sciences. Behavior Research Methods 2007; 39 (2): 175-91.

14. O'brien E, Atkins N, Stergiou G, Karpettas N, Parati G, Asmar R, et al. European Society of Hypertension International Protocol revision 2010 for the validation of blood pressure measuring devices in adults. Blood Press Monit 2010; 15 (1): 23-38.

15. Lim Y-H, Choi SY, Oh KW, Kim Y, Cho ES, Choi BY, et al. Comparison between an automated device and a manual mercury sphygmomanometer in an epidemiological survey of hypertension prevalence. Am J Hypertens 2013; 27 (4): 537-45. 